\title{
Confinement of Antiprotons in the Electrostatic Space Charge of Positrons in a Model of the ALPHA Antihydrogen Trap
}

\author{
R. A. Lane and C. A. Ordonez $\ddagger$ \\ Department of Physics, University of North Texas, Denton, Texas 76203 \\ E-mail: cao@unt.edu
}

\begin{abstract}
The equilibrium of a positron plasma in a model of the ALPHA apparatus is computed using a finite-difference method. The positron plasma in the model extends to axial magnetic mirrors in absence of an octupole field. Formation of a three-dimensional electrostatic potential well is found to occur self-consistently. Well depths under various conditions are evaluated. Also, the equilibrium with an antiproton plasma confined in the potential well is computed. Conditions from the two species case are used to estimate timescales associated with three-body recombination, antiproton loss, and temperature equilibration. An equilibrium where the three-body recombination timescale is the smallest of the three is identified.
\end{abstract}

PACS numbers: 41.20.Cv, 41.20.Gz, 52.55.Jd, 52.27.Jt

antihydrogen, space charge, plasma equilibrium, finite difference

Submitted to: J. Phys. B: At. Mol. Opt. Phys.

$\ddagger$ Author to whom correspondence should be addressed. 


\section{Introduction}

Multiple collaborations including ALPHA [1, 2], ATRAP [3, 4], ASACUSA [5, 6], AEGIS $[7,8]$, and GBAR [9] propose to use antihydrogen for tests of CPT (charge conjugation, parity, time-reversal) and/or gravitational symmetries. Successful production and confinement of antihydrogen on timescales of up to $10^{3} \mathrm{~s}$ have been demonstrated $[4,10]$. Current experiments involve relatively small numbers of antiatoms and future experiments may benefit from increases in production of trappable antihydrogen $[11,12,13]$.

The ALPHA and ATRAP experiments aim to confine antihydrogen atoms in traps based on the Ioffe-Pritchard configuration [3, 14, 15]. Charged particles are confined in a Malmberg-Penning trap that has additional magnetic fields superimposed to create a magnetic minimum for neutral particle confinement. The minimum field consists of the superposition of a uniform field, a transverse multipole field, and axial mirror fields. Antihydrogen producing plasmas are confined near the center of the trap, where the multipole and axial mirror fields are weak relative to the uniform field.

In the ALPHA experiment, potentials applied to the Malmberg-Penning trap electrodes create axial nested potential wells that simultaneously confine antiprotons and positrons. Autoresonant excitation of the antiproton plasma is used to mix the two species $[16,17]$. For antihydrogen in a low-field seeking state, confinement occurs through interaction of an antihydrogen atom's intrinsic magnetic moment with the magnetic minimum field.

In the present study, equilibria of non-neutral plasmas are evaluated in a model of the ALPHA trap. Malmberg-Penning trap electrode potentials produce a single well such that the positron plasma extends axially to the magnetic mirrors in the absence of the transverse multipole field. By extending to higher magnetic field regions, the positron plasma self-consistently produces a three-dimensional potential well. The formation of electric potential wells in Penning traps with mirror fields has been discussed previously $[18,19]$. Conditions leading to potential well formation in a positron plasma are used in the present study to investigate the possibility of simultaneous confinement of two plasma species.

Equilibria are calculated by self-consistently solving Poisson's equation using a finite-difference computational approach [20]. Computational techniques are developed to apply the Boltzmann density relation along curved magnetic field lines. Once developed, the techniques are applied to calculate the equilibrium of a positron plasma in the model trap. Two-species equilibria are also evaluated for an antiproton plasma that follows the Boltzmann density distribution in three dimensions and is confined by the space charge of a positron plasma. Equilibria of non-neutral plasmas have been previously computed using similar methods in multiple trap geometries, but with uniform magnetic fields or no magnetic field [21, 22, 23].

The equilibrium solutions presented here require the Boltzmann density relation to hold along each magnetic field line for positron plasmas. The theoretical treatment 
considers the positron plasma to extend axially to each magnetic mirror coil, where a boundary of the computation region is located. Thus, the theoretical treatment doesn't include axial positron confinement, aside from that associated with the magnetic mirror effect on the positron density distribution. For a real system, an electric field could be applied just beyond each magnetic mirror coil, such that the positron plasma extends axially to each mirror coil but not farther. The associated electric potential well could be made deep enough for axial positron losses to be negligible.

The theoretical treatment also doesn't include radial positron confinement, aside from assuming that positrons follow magnetic field lines. Although radial plasma transport processes are considered to be beyond the scope of the present study, an important effect of radial transport processes should be mentioned. Radial expansion of the positron plasma could convert potential energy to kinetic energy thereby heating the plasma. Collisions among particles can cause radial plasma transport processes to occur. Because a non-neutral plasma rotates, a magnetic field that deviates from being cylindrically symmetric can cause radial plasma transport processes to occur. The octupole field causes such a deviation. In addition to affecting radial transport processes, the octupole field may directly affect plasma equilibria. A self-consistent three-dimensional computation would be needed for obtaining plasma equilibria that include the octupole field. For connecting the present theoretical work with a real system, it must be kept in mind that a limitation of the present work is that the computation is two-dimensional and neglects effects associated with the presence of the octupole field.

The model of the ALPHA trap is described in section 2, and the computational techniques are detailed in section 3 and section 4. Equilibria showing potential well formation in a positron plasma are presented in section 5, and equilibria for an antiproton plasma confined in the potential well created by the space charge of a positron plasma are reported in section 6 . The three-body recombination timescale, antiproton loss timescale, and temperature equilibration timescale are calculated for two-species equilibria in section 7. The computations are discussed in section 8 and a summary is given in section 9. Throughout, subscripts + and - designate quantities that pertain only to positrons and antiprotons, respectively.

\section{Model}

The model of the ALPHA trap is illustrated in figure 1 and consists of a MalmbergPenning trap with magnetic mirror fields. The magnitude of the transverse octupole magnetic field near the radial center of the trap where the plasma is located is assumed to be negligible in the model. Mirror fields are represented by the magnetic fields of two circular current loops centered on the $z$ axis at $z= \pm z_{\mathrm{m}}$, and the uniform magnetic field is $\boldsymbol{B}_{\mathrm{U}}=B_{\mathrm{U}} \hat{\boldsymbol{z}}$. The superposition principle gives the two components of the total magnetic field in cylindrical coordinates as

$$
B_{\mathrm{r}}(r, z)=B_{\mathrm{m}, \mathrm{r}}\left(r, z-z_{\mathrm{m}}\right)
$$


Confinement of $\bar{p}$ in $e^{+}$space charge

$$
\begin{aligned}
& +B_{\mathrm{m}, \mathrm{r}}\left(r, z+z_{\mathrm{m}}\right), \\
B_{\mathrm{z}}(r, z) & =B_{\mathrm{m}, \mathrm{z}}\left(r, z-z_{\mathrm{m}}\right) \\
& +B_{\mathrm{m}, \mathrm{z}}\left(r, z+z_{\mathrm{m}}\right)+B_{\mathrm{U}} .
\end{aligned}
$$

The components $B_{\mathrm{m}, \mathrm{r}}(r, z)$ and $B_{\mathrm{m}, \mathrm{z}}(r, z)$ are given in the Appendix.
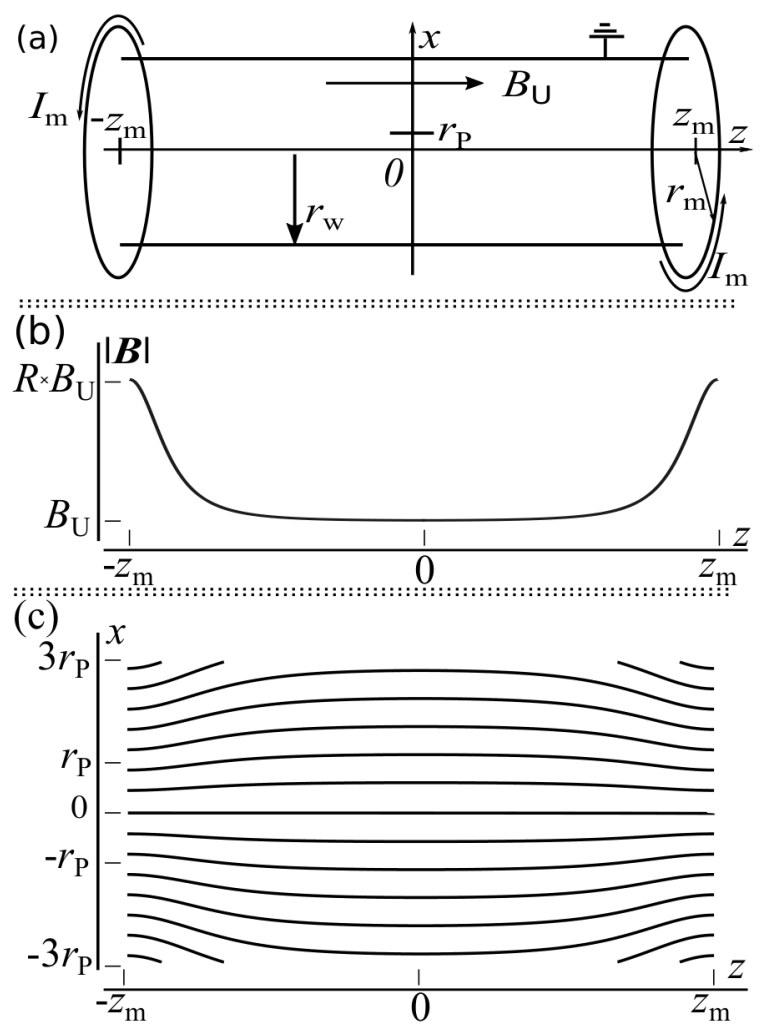

Figure 1. A schematic of the model for the ALPHA trap is shown in (a), the magnetic field magnitude along the $z$ axis is plotted in (b), and magnetic field lines are shown in (c). The model is symmetric about the $z=0$ plane and is cylindrically symmetric. In (b), $R$ is the mirror ratio along the $z$ axis.

The model is radially bounded by a grounded cylindrical electrode centered on the $z$ axis with inner radius $r_{\mathrm{w}}$. The electrostatic potential profile is computed in the region $0 \leq r \leq r_{\mathrm{w}},-z_{\mathrm{m}} \leq z \leq 0$ and applied to other regions through symmetry. Neumann boundary conditions are set at $z=0, r=0$, and in the $z=-z_{\mathrm{m}}$ plane. Boundary conditions are consistent with symmetry about the $z=0$ plane, cylindrical symmetry, and approximate symmetry near the $z=-z_{\mathrm{m}}$ plane. The Neumann boundary condition in the $z=-z_{\mathrm{m}}$ plane closes the otherwise open boundary. Symbolically, the boundary conditions are

$$
\begin{aligned}
& \phi\left(r_{\mathrm{w}}, z\right)=0, \\
& \left.\frac{\partial \phi(r, z)}{\partial r}\right|_{r=0}=0, \\
& \left.\frac{\partial \phi(r, z)}{\partial z}\right|_{z=0}=0
\end{aligned}
$$


Confinement of $\bar{p}$ in $e^{+}$space charge

$$
\left.\frac{\partial \phi(r, z)}{\partial z}\right|_{z=-z_{\mathrm{m}}}=0
$$

where $\phi(r, z)$ is the electric scalar potential.

\section{Methods}

Equilibria are computed by solving Poisson's equation, $\nabla^{2} \phi(\boldsymbol{r})=-\rho(\boldsymbol{r}) / \epsilon_{0}$, through a self-consistent finite-difference computation [20]. Here, $\rho(\boldsymbol{r})$ is the charge density and $\epsilon_{0}$ is the vacuum permittivity. The charge density for a confined positron plasma can be written as $\rho(\boldsymbol{r})=e n_{+}(\boldsymbol{r})$, where $n_{+}(\boldsymbol{r})$ is the positron density distribution, each positron carrying elementary charge $e$.

The positron density in the $z=0$ plane is considered to be radially uniform out to a radius $r=r_{\mathrm{P}}$, where it possesses a sharp edge. Symbolically, the density in the $z=0$ plane is $n_{+}(r, 0)=\Theta\left(r_{\mathrm{P}}-r\right) n_{0+}$, where $\Theta$ is the Heaviside step function defined as $\Theta(x<0)=0, \Theta(x \geq 0)=1$ and $n_{0+}$ is the positron density. If the positron plasma follows the Boltzmann density relation along magnetic field lines, the density at any point is

$$
\begin{aligned}
n_{+}(r, z) & =n_{0+} \Theta\left(r_{\mathrm{P}}-r_{0}\right) \\
& \times \exp \left(-\frac{e}{k_{\mathrm{B}} T_{+}}\left[\phi(r, z)-\phi\left(r_{0}, 0\right)\right]\right),
\end{aligned}
$$

where $T_{+}$is the positron plasma temperature, $k_{\mathrm{B}}$ is the Boltzmann constant, and $r_{0}$ is the radial position where the magnetic field line passing through $(r, z)$ intersects the $z=0$ plane. Magnetic field lines are solutions to $\mathrm{d} r / B_{\mathrm{r}}(r, z)=\mathrm{d} z / B_{\mathrm{z}}(r, z)$, which is solved numerically in parametric form,

$$
\begin{aligned}
\frac{\mathrm{d} r}{\mathrm{~d} s} & =\frac{B_{\mathrm{r}}(r(s), z(s))}{|\boldsymbol{B}(r(s), z(s))|}, \\
\frac{\mathrm{d} z}{\mathrm{~d} s} & =\frac{B_{\mathrm{z}}(r(s), z(s))}{|\boldsymbol{B}(r(s), z(s))|},
\end{aligned}
$$

using a fourth-order Rungge-Kutta solver.

\section{Computations}

A finite-difference approximation with central differencing starting from an arbitrary initial solution $\phi^{(0)}(r, z)$ is used to self-consistently solve Poisson's equation. The potential is calculated at the vertices of a computational mesh with radial spacing $\Delta r$ and horizontal spacing $\Delta z$. Values for each iteration are obtained through

$$
\begin{aligned}
& \phi^{(l+1)}(r, z)=-(\alpha-1) \phi^{(l)}(r, z) \\
& +\frac{\alpha}{2}\left(\frac{1}{\Delta r^{2}}+\frac{1}{\Delta z^{2}}\right)^{-1} \\
& \quad \times\left[\frac{\phi^{(l)}(r+\Delta r, z)-\phi^{(l)}(r-\Delta r, z)}{2 r \Delta r}\right.
\end{aligned}
$$


Confinement of $\bar{p}$ in $e^{+}$space charge

$$
\begin{aligned}
& +\frac{\phi^{(l)}(r+\Delta r, z)+\phi^{(l)}(r-\Delta r, z)}{\Delta r^{2}} \\
& \left.+\frac{\phi^{(l)}(r, z+\Delta z)+\phi^{(l)}(r, z-\Delta z)}{\Delta r^{2}}-f^{(l)}(r, z)\right],
\end{aligned}
$$

where, $\alpha$ is a mixing constant taking values $0<\alpha \leq 1$ and $f(r, z)=-\rho(r, z) / \epsilon_{0}$. Here, $\rho=e n_{+}$is the charge density, and equation (3) is used to calculate the positron density $n_{+}$. A review of iterative finite-difference techniques can be found in [20].

Generally, mesh spacing of less than half the Debye length is needed for convergence [20]. In the model, the criteria $\Delta r<\lambda_{\mathrm{D}+} / 4$ and $\Delta z<\lambda_{\mathrm{D}+} / 4$ are used. Here, an additional minimum factor of two is introduced to offset increased density expected in the high field region [18], and $\lambda_{\mathrm{D}+}$ is the Debye length for a positron plasma with temperature $T_{+}$and density $n_{0+}$ given by $\lambda_{\mathrm{D}+}=\left[\epsilon_{0} k_{\mathrm{B}} T_{+} /\left(e^{2} n_{0+}\right)\right]^{1 / 2}$. For example, for a $40 \mathrm{~K}$ plasma with density $5.5 \times 10^{13} \mathrm{~m}^{-3}, \lambda_{\mathrm{D}+}=5.9 \times 10^{-5} \mathrm{~m}$. A method to reduce computation time is employed.

The region that requires mesh spacing dictated by the Debye length is known a priori to be $r \lesssim r_{\mathrm{P}}$. With $r_{\mathrm{P}} \ll r_{\mathrm{w}}$, computation time is reduced by employing two uniform mesh spacings where the coarse spacing is a multiple of the fine spacing. The static mesh refinement method employed here is developed from adaptive mesh refinement methods [24] by using simplifying assumptions. Mesh spacing is $\Delta r_{\mathrm{A}}=$ $\Delta z_{\mathrm{A}}=10^{-5} \mathrm{~m}$ in the region $r \leq 2 r_{\mathrm{P}}$ and $\Delta r_{\mathrm{B}}=\Delta z_{\mathrm{B}}=10^{-4} \mathrm{~m}$ in the region $r>2 r_{\mathrm{P}}$ and is sketched in figure 2 .

The potentials $\phi^{(l+1)}\left(r<2 r_{\mathrm{P}}, z\right)$ and $\phi^{(l+1)}\left(r>2 r_{\mathrm{P}}, z\right)$ are calculated through direct application of (5). Calculations for potentials with $r=2 r_{\mathrm{P}}$ use 'ghost' points along the line $r=2 r_{\mathrm{P}}+\Delta r_{\mathrm{A}}$ for the forward difference terms. For example, a ghost point is located at $\left(2 r_{\mathrm{P}}+\Delta r_{\mathrm{A}}, z_{\mathrm{A}}\right)$ in figure 2. The potential at $\left(2 r_{\mathrm{P}}+\Delta r_{\mathrm{A}}, z_{\mathrm{A}}\right)$ is calculated through linear interpolation of $\phi^{(l)}\left(2 r_{\mathrm{P}}+\Delta r_{\mathrm{B}}, z_{\mathrm{A}}\right)$ and $\phi^{(l)}\left(2 r_{\mathrm{P}}, z_{\mathrm{A}}\right)$. Interpolation from axially adjacent points is used when $\left(2 r_{\mathrm{P}}+\Delta r_{\mathrm{B}}, z_{\mathrm{A}}\right)$ lies between previously calculated values.

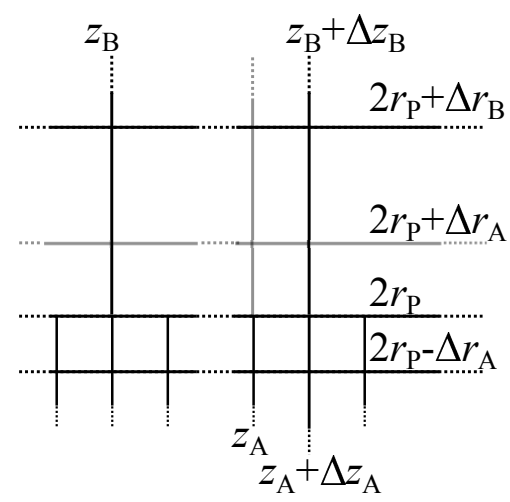

Figure 2. The computational grid used consists of two regions. The region with $r \leq 2 r_{\mathrm{P}}$ has points spaced at $\Delta r_{\mathrm{A}}=\Delta z_{\mathrm{A}}$ and the region at $r>2 r_{\mathrm{P}}$ uses spacing $\Delta r_{\mathrm{B}}=\Delta z_{\mathrm{B}}$ with $\Delta r_{\mathrm{B}}=m \Delta r_{\mathrm{B}}$ where $m$ is an integer. 


\section{Equilibria Conditions}

Model dimensions are selected to represent the ALPHA trap [25]. In the model, Malmberg-Penning trap electrodes have inner radius $r_{\mathrm{w}}=22.3 \mathrm{~mm}$, and the uniform magnetic field is $B_{\mathrm{U}}=1.0 \mathrm{~T}$. The current loops have radius $r_{\mathrm{m}}=44.5 \mathrm{~mm}$ and are centered at $(0,13.7 \mathrm{~cm})$ and $(0,-13.7 \mathrm{~cm})$. In a 'base case' configuration, parameters are selected to represent feasible conditions in the ALPHA trap [1, 2]. The mirror coils produce a total magnetic field magnitude at $(0, \pm 13.7 \mathrm{~cm})$ of $2.0 \mathrm{~T}$, giving a mirror ratio along the $z$ axis of $R=2.0$. The positron plasma is set to a temperature of $T_{+}=40 \mathrm{~K}$ and has a uniform density in the $z=0$ plane $n_{0+}=5.5 \times 10^{13} \mathrm{~m}^{-3}$ out to radius $r_{\mathrm{P}}=0.9 \mathrm{~mm}$.

The axial potential well is the difference between the maximum and minimum potentials along the $z$ axis, while the radial potential well is the difference between the maximum potential and the electrode applied potential. A contour plot of the electrostatic potential near the $z$ axis is shown in figure 3. Plots of the positron density are shown in figure 4 . For the base case, the radial potential well depth is $6.7 \times 10^{3} \mathrm{~K}$ and the axial potential well depth is $27 \mathrm{~K}$. The possibility of using the electric potential well to simultaneously confine antiprotons is explored in section 6 .

Values for the magnetic field mirror ratio, positron density, plasma radius, and plasma temperature are individually changed from the base case. Axial and radial potential well depths under each change are given in table 1 . The base case is shown at the top of the table, and parameter values that deviate from the base case are given in the first two columns for other cases. Increases in the positron plasma temperature or the axial mirror ratio lead to an increased axial potential well depth while leaving the radial potential well approximately unchanged. Changes to the positron density and plasma radius affect the radial well depths with small changes to the axial well depth. The well depths are converted to temperature units through $U_{\mathrm{W}}=e \Delta \phi / k_{\mathrm{B}}$.

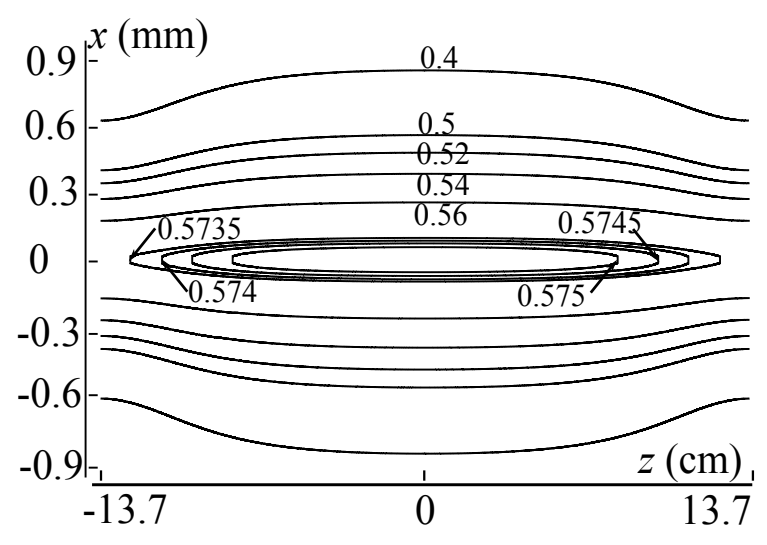

Figure 3. A contour plot of the computed electrostatic potential (in volts) within a confined positron plasma. The results indicate the formation of a three-dimensional electric potential well, which may serve for simultaneously confining antiprotons. 
Table 1. Potential well depth under various conditions in a model of the ALPHA trap. The well depth is converted to temperature units through $U_{\mathrm{W}}=e \Delta \phi / k_{\mathrm{B}}$. The base case has $r_{\mathrm{P}}=0.9 \mathrm{~mm}, T_{+}=40 \mathrm{~K}, n_{0+}=5.5 \times 10^{13} \mathrm{~m}^{-3}$, and $R=2.0$. Parameters are individually varied from base case conditions. The symbol for the parameter varied is listed in the first column and the parameter value is given in the second column. The fifth column gives the ratio of the positron density at $r=0$ in the plane of the mirror coil to the density at the center of the trap. The final column gives the full width at half maximum (FWHM) density for the positron plasma in the the $z=-z_{\mathrm{m}}$ plane.

\begin{tabular}{|c|c|c|c|c|c|}
\hline \multicolumn{2}{|r|}{ parameter } & $U_{\mathrm{W}}($ radial $)$ & $U_{\mathrm{W}}($ axial $)$ & $n_{+}\left(0,-z_{\mathrm{m}}\right) / n_{0+}$ & FWHM \\
\hline symbol & value & $10^{3} \mathrm{~K}$ & $\mathrm{~K}$ & unitless & $\mathrm{mm}$ \\
\hline \multicolumn{2}{|r|}{ base } & 6.68 & 26.8 & 1.95 & 1.14 \\
\hline$T_{+}$ & $20 \mathrm{~K}$ & 6.69 & 13.6 & 1.97 & 1.14 \\
\hline$T_{+}$ & $80 \mathrm{~K}$ & 6.67 & 53.1 & 1.94 & 1.16 \\
\hline$n_{0+}$ & $1.0 \times 10^{13} \mathrm{~m}^{-3}$ & 1.43 & 27.9 & 2.01 & 1.14 \\
\hline$n_{0+}$ & $1.0 \times 10^{14} \mathrm{~m}^{-3}$ & 11.5 & 26.4 & 1.93 & 1.16 \\
\hline$r_{\mathrm{P}}$ & $0.5 \mathrm{~mm}$ & 2.30 & 26.7 & 1.95 & 0.66 \\
\hline$r_{\mathrm{P}}$ & $1.3 \mathrm{~mm}$ & 12.5 & 25.8 & 1.91 & 1.66 \\
\hline $\mathrm{R}$ & 1.5 & 6.68 & 15.7 & 1.48 & 1.40 \\
\hline $\mathrm{R}$ & 3.0 & 6.68 & 41.1 & 2.79 & 0.84 \\
\hline
\end{tabular}
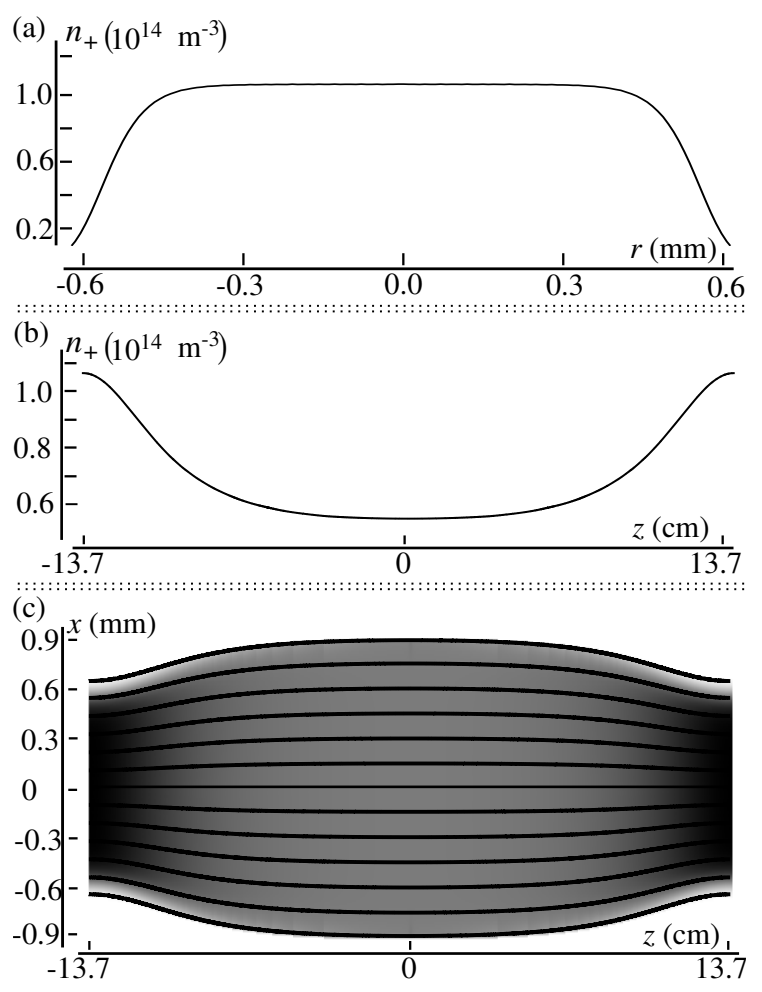

Figure 4. The equilibrium positron density distribution is shown for the mirror plane $(z=-13.7 \mathrm{~cm})$ in (a) and along the $z$ axis in (b). In (c), the equilibrium positron distribution in two dimensions is shown with magnetic field lines. Darker regions indicate higher densities and magnetic field lines are depicted as solid black lines. 


\section{Two-Species Confinement}

For two-species confinement, the electric potential profile is computed from Poisson's equation by including the distribution of both species through $\rho(\boldsymbol{r})=e\left[n_{+}(\boldsymbol{r})-n_{-}(\boldsymbol{r})\right]$. Here, the positron density distribution, $n_{+}(\boldsymbol{r})$, is given by (3) and $n_{-}(\boldsymbol{r})$ is the antiproton density distribution. Antiprotons are assumed to be confined in the potential well created by the positron plasma and to follow the Boltzmann density distribution in three dimensions. The antiproton density at the coordinate origin is specified as $n_{0-}$ and the density elsewhere is given by

$$
n_{-}(r, z)=n_{0-} \exp \left(\frac{e[\phi(r, z)-\phi(0,0)]}{k_{\mathrm{B}} T_{-}}\right),
$$

where $T_{-}$is the antiproton temperature.

In the two-species base case, the antiprotons are at $4 \mathrm{~K}$ and have density $n_{0-}=$ $1.0 \times 10^{12} \mathrm{~m}^{-3}$, and the positron plasma parameters are the same as in the single-species base case. The two-species base case equilibrium is found to possess a radial well depth of $6.6 \times 10^{3} \mathrm{~K}$ and an axial well depth of $27 \mathrm{~K}$. Compared to the single-species base case, the axial and radial well depths are approximately unchanged. The equilibrium antiproton density under two-species base case conditions is shown in figure 5 .
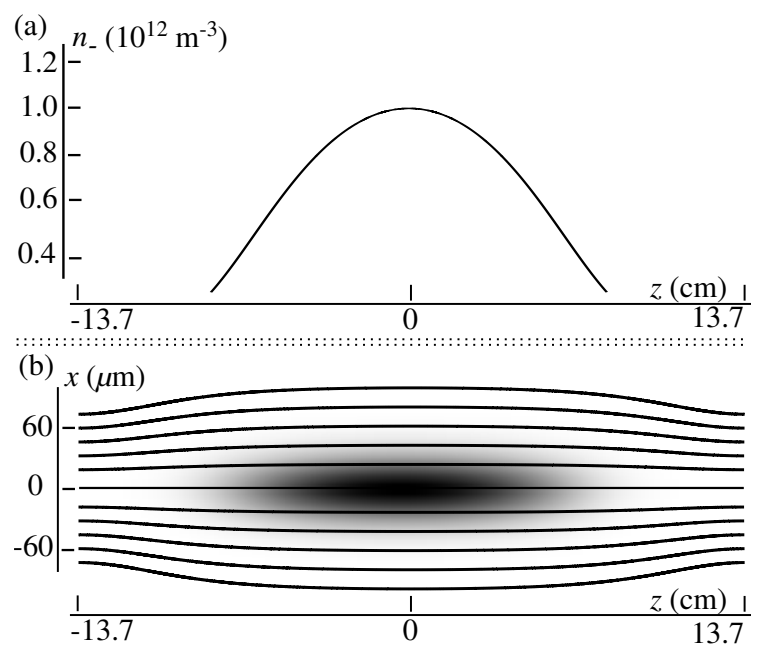

Figure 5. The equilibrium antiproton density distribution along the $z$ axis is shown in (a). In (b), the antiproton density near the center of the trap is shown with magnetic field lines. Darker regions indicate higher density and magnetic field lines are shown as solid, black lines.

The well depths and antiproton plasma dimensions under various conditions are reported in table 2. Values for the two-species base case are given in the first row. Parameters are individually varied from the two-species base case and the parameter that is varied and its value are given in the first two columns. The full width at half the maximum (FWHM) density for the antiproton plasma is given radially at $z=0$ and along the $z$ axis (axial) in the final two columns. 
Table 2. Well depths and the dimensions of the antiproton plasma are given under various conditions. The two-species base case conditions are $r_{P}=0.9 \mathrm{~mm}, T_{+}=40 \mathrm{~K}$, $T_{-}=4 \mathrm{~K}, n_{0+}=5.5 \times 10^{13} \mathrm{~m}^{-3}, n_{0-}=1.0 \times 10^{12} \mathrm{~m}^{-3}$, and $R=2.0$. Parameters are individually changed from the base case. The symbol for the parameter changed and its value are given in the first two columns. The full width of the antiproton plasma at half its maximum density (FWHM) is given radially at $z=0$ and along the $z$ axis (axial).

\begin{tabular}{cccccc}
\hline \multicolumn{2}{c}{$\begin{array}{c}\text { parameter } \\
\text { symbol }\end{array}$} & $\begin{array}{c}U_{\mathrm{W}}(\text { radial }) \\
10^{3} \mathrm{~K}\end{array}$ & $U_{\mathrm{W}}$ (axial) & FWHM (radial) & FWHM (axial) \\
& base & 6.65 & 26.6 & 100 & $\mathrm{Km}$ \\
$T_{+}$ & $20 \mathrm{~K}$ & 6.63 & 13.5 & 100 & 11.8 \\
$T_{+}$ & $80 \mathrm{~K}$ & 6.63 & 52.9 & 100 & 15.0 \\
$T_{-}$ & $0.4 \mathrm{~K}$ & 6.63 & 26.7 & 60 & 8.94 \\
$T_{-}$ & $8 \mathrm{~K}$ & 6.63 & 26.6 & 120 & 4.33 \\
$n_{0+}$ & $1.0 \times 10^{13} \mathrm{~m}^{-3}$ & 1.42 & 27.1 & 180 & 15.1 \\
$n_{0+}$ & $1.0 \times 10^{14} \mathrm{~m}^{-3}$ & 11.4 & 26.3 & 80 & 12.1 \\
$n_{0-}$ & $5.0 \times 10^{11} \mathrm{~m}^{-3}$ & 6.63 & 26.7 & 100 & 11.8 \\
$n_{0-}$ & $5.0 \times 10^{12} \mathrm{~m}^{-3}$ & 6.63 & 26.0 & 100 & 11.7 \\
$r_{P}$ & $0.5 \mathrm{~mm}$ & 2.27 & 26.5 & 100 & 12.3 \\
$r_{P}$ & $1.3 \mathrm{~mm}$ & 12.4 & 25.6 & 100 & 11.8 \\
$R$ & 1.5 & 6.65 & 15.6 & 100 & 12.0 \\
$R$ & 3.0 & 6.65 & 40.9 & 100 & 9.20 \\
\hline
\end{tabular}

In some cases, the radial FWHM density for the antiproton plasma is less than $10 \Delta r_{\mathrm{A}}$. An uncertainty from mesh size, of $\pm 5 \mu \mathrm{m}$ is associated with the values. Varying the parameters of the positron plasma produces changes in the well depths similar to the single-species cases. Equilibria computed with the density or temperature of the antiproton plasma varied, have axial and radial potential well depths within $5 \%$ of the two-species base case value. With these variations, equilibrium antiproton FWHM dimensions deviate from the two-species base case by up to $40 \%$ radially and $63 \%$ axially.

\section{Timescale Calculations}

The three-body recombination process that produces stable Rydberg atoms can be associated with the timescale [26]

$$
\tau_{\mathrm{TBR}} \approx 1.8 \times 10^{21} \frac{T_{+}^{9 / 2}}{n_{+}^{2}} .
$$

Collisions between antiprotons can cause losses from an effectively one-dimensional electrostatic well. An expression for the axial loss rate for plasma particles in an electric potential well and a uniform magnetic field is obtained in [27] using the axial loss rate of a plasma in a magnetic mirror field with an electric potential well derived in [28]. 
The axial loss rate of antiprotons is estimated as [27]

$$
\begin{aligned}
\dot{n}_{-} & =\frac{0.0059 \lambda\left(e^{2} n_{0-}\right)^{2}}{\sqrt{\epsilon_{0}^{4} M_{-}\left(k_{\mathrm{B}} T_{-}\right)^{3}}} \\
& \times\left(\frac{\mathrm{e}^{-\omega}}{\omega}+\sqrt{\frac{\pi}{4 \omega^{3}}} \operatorname{erfc}(\sqrt{\omega})\right) .
\end{aligned}
$$

Here, all dimensionless constants are reduced to a numerical factor, $\dot{n}_{-}$is the rate of change of the antiproton density, $M_{-}$is the antiproton mass, $\omega=U_{\mathrm{W}} / T_{-}=e \Delta \phi /\left(k_{\mathrm{B}} T_{-}\right)$ is the unitless ratio of the electrostatic potential energy well depth to the antiproton temperature, erfc is the complimentary error function, and $\lambda$ is the Coulomb logarithm. The timescale associated with antiproton loss is calculated as $\tau_{-}=n_{0-} / \dot{n}_{-}$.

The Coulomb logarithm is calculated through $[29,30]$

$$
\lambda=\frac{(1+\Lambda)^{2} \ln \left[(1+\Lambda)^{2}\right]}{2(2+\Lambda)^{2}}-\frac{\Lambda}{2(2+\Lambda)},
$$

where $\Lambda=\rho_{\max } / \beta$. For Coulomb collisions between antiprotons, the impact parameter for $90^{\circ}$ scattering in the center-of-mass frame is $\beta=e^{2}\left(8 \pi \epsilon_{0} k_{\mathrm{B}} T_{-}\right)^{-1}$ [27], and the maximum binary interaction radius is taken to be $\rho_{\max }=\min \left(\lambda_{\mathrm{D}+}, \lambda_{\mathrm{D}-}, 2 r_{\mathrm{c}-}\right)$. Here min returns the smallest of its argument's values, $\lambda_{\mathrm{D}-}=\left[\epsilon_{0} k_{\mathrm{B}} T_{-} /\left(e^{2} n_{0_{-}}\right)\right]^{1 / 2}$ is the Debye length associated with an antiproton plasma with density $n_{0-}$ at the center of the trap, and $r_{\mathrm{c}-}=\left(M_{-} k_{\mathrm{B}} T_{-} B^{-2} e^{-2}\right)^{1 / 2}$ is the cyclotron radius of an antiproton with mass $M_{-}$moving in a uniform magnetic field with magnitude $B$.

The effect of the magnetic field is not accounted for except, when the antiproton cyclotron radius is sufficiently small, $\rho_{\max }=2 r_{\mathrm{c}-}$ is used. The collision theory used for the calculation of the Coulomb logarithm is known to not be valid for binary collisions involving particles with like charges when $\lambda<0.06$ [30]. The Coulomb logarithm values are not affected by this limit for the equilibria considered in table 2 except the case where $T_{-}=0.4 \mathrm{~K}$.

When simultaneously confined, a two-species plasma with disparate temperatures will equilibrate through Coulomb collisions between particles. For an antiproton and positron plasma, the equilibration timescale is calculated by [31]

$$
\begin{aligned}
\tau_{\mathrm{EQ}} & =\frac{47.2 \epsilon_{0}^{2} M_{-} M_{+}}{e^{4} n_{0+} \Gamma\left(0, \frac{4}{\pi} \mathrm{e}^{-2 \lambda}, \frac{4}{\pi}\right)} \\
& \times\left(\frac{k_{\mathrm{B}} T_{-}}{M_{-}}+\frac{k_{\mathrm{B}} T_{+}}{M_{+}}\right)^{3 / 2} .
\end{aligned}
$$

Here, $\Gamma$ is the zeroth-order incomplete gamma function. The Coulomb logarithm in (10) is calculated through (9). For Coulomb collisions between an antiproton and a positron $\beta=-e^{2}\left[32 \epsilon_{0} k_{\mathrm{B}}\left(T_{+} M_{-}+T_{-} M_{+}\right) /\left(M_{+}+M_{-}\right)\right]^{-1}$ is used [31], where the leading sign is incorporated to account for the opposite charge signs of the colliding particles. The maximum binary interaction radius is taken to be $\rho_{\max }=\min \left(\lambda_{\mathrm{D}+}, \lambda_{\mathrm{D}-}, 2 r_{\mathrm{c}-}, 2 r_{\mathrm{c}+}\right)$, which includes the cyclotron radius for a positron moving in a uniform magnetic field with magnitude $B, r_{\mathrm{c}+}=\left[M_{+} k_{\mathrm{B}} T_{+} B^{-2} e^{-2}\right]^{1 / 2}$. 
Table 3. Timescales associated with three-body recombination $\left(\tau_{\mathrm{TBR}}\right)$, losses due to antiproton-antiproton collisions $\left(\tau_{-}\right)$, and temperature equilibration between the antiprotons and the positrons $\left(\tau_{\mathrm{EQ}}\right)$ are calculated for the two-species plasma.

\begin{tabular}{ccccc}
\hline \multicolumn{2}{c}{ parameter } & $\tau_{\mathrm{TBR}}$ & $\tau_{-}$ & $\tau_{\mathrm{EQ}}$ \\
symbol & value & $\mathrm{s}$ & $\mathrm{s}$ & $\mathrm{ms}$ \\
& base & 9.63 & 5.02 & 2.26 \\
$T_{+}$ & $20 \mathrm{~K}$ & 0.426 & 0.0899 & 8.77 \\
$T_{+}$ & $80 \mathrm{~K}$ & 217 & $>10^{3}$ & 2.85 \\
$T_{-}$ & $0.4 \mathrm{~K}$ & 9.63 & $>10^{3}$ & 2.26 \\
$T_{-}$ & $8 \mathrm{~K}$ & 9.63 & 0.0790 & 2.26 \\
$n_{0+}$ & $1.0 \times 10^{13} \mathrm{~m}^{-3}$ & 291 & 5.75 & 12.4 \\
$n_{0+}$ & $1.0 \times 10^{14} \mathrm{~m}^{-3}$ & 2.91 & 4.52 & 1.24 \\
$n_{0-}$ & $5.0 \times 10^{11} \mathrm{~m}^{-3}$ & 9.63 & 10.3 & 2.26 \\
$n_{0-}$ & $5.0 \times 10^{12} \mathrm{~m}^{-3}$ & 9.63 & 0.833 & 2.26 \\
$r_{\mathrm{P}}$ & $0.5 \mathrm{~mm}$ & 9.63 & 4.85 & 2.26 \\
$r_{\mathrm{P}}$ & $1.3 \mathrm{~mm}$ & 9.63 & 3.78 & 2.26 \\
$R$ & 1.5 & 9.63 & 0.178 & 2.26 \\
$R$ & 3.0 & 9.63 & 284 & 2.26
\end{tabular}

The timescales associated with the equilibria described in table 2 are given in table 3. For the two-species base case $\tau_{\mathrm{TBR}}=9.6 \mathrm{~s}, \tau_{-}=5.0 \mathrm{~s}$, and $\tau_{\mathrm{EQ}}=2.3 \mathrm{~ms}$. For the equilibria in table 2 , the temperature equilibration timescale is much smaller than the three-body recombination timescale. In (7), the three-body recombination timescale depends strongly on the positron temperature and, in the parameter space considered, decreases to less than $1 \mathrm{~s}$ when the positron temperature is reduced to $20 \mathrm{~K}$.

The timescale associated with losses due to antiproton-antiproton collisions varies under each parameter set with deviations of more than two orders of magnitude from the timescale with two-species base case conditions. Timescales for temperature equilibration between the positrons and antiprotons demonstrate weaker dependence on the parameters considered. For $\tau_{E Q}$, the greatest deviation from two-species base case conditions is less than an order of magnitude.

Experimental conditions where $\tau_{\mathrm{TBR}}<\tau_{\mathrm{EQ}}$ and $\tau_{\mathrm{TBR}}<\tau_{-}$are preferable for antihydrogen trapping. Equilibria meeting these conditions can be computed. By way of example, an equilibrium is found for a two-species plasma with $T_{+}=5 \mathrm{~K}, T_{-}=0.5 \mathrm{~K}$, $n_{0+}=2.0 \times 10^{13} \mathrm{~m}^{-3}, n_{0-}=2.0 \times 10^{11} \mathrm{~m}^{-3}, r_{P}=0.9 \mathrm{~mm}$, and $R=2.0$. This equilibrium has an axial potential well depth of $3.6 \mathrm{~K}$, yielding timescales $\tau_{\mathrm{TBR}}=6.3 \mathrm{~ms}, \tau_{-}=410 \mathrm{~s}$, and $\tau_{\mathrm{EQ}}=340 \mathrm{~ms}$. A revised computation of $\tau_{-}$is called for as $\lambda<0.06$ occurs.

\section{Discussion}

Using the space charge of the positron plasma to confine or enhance confinement of the antiprotons in an Ioffe-Pritchard trap represents an alternative approach to antihydrogen production. Some trends are reported here in the potential well depths, plasma dimensions, and various timescales under variation of a few parameters. Detailed 
investigations using the finite-difference methods employed here and particle-in-cell simulations such as those used in [19] could serve to further probe the relationships between the plasma parameters and equilibrium conditions.

The theoretical approach used to compute equilibria neglects effects associated with plasma rotation and does not simulate the time-dependent approach to the equilibria. For all equilibria computed, iterations were continued until the maximum change between iterations at any grid point was less than $5.0 \times 10^{-7} \mathrm{~V}$. The positron density distribution obtained for the base case (figure 4) is consistent with analytical studies [18] and particle-in-cell studies [19] of non-neutral plasmas in non-uniform magnetic fields.

The temperature equilibration timescales in table 3 are smaller than the threebody recombination timescales. The parameters that lead to $\tau_{\mathrm{TBR}}<\tau_{\mathrm{EQ}}$ require colder positron plasmas. Using the electrostatic space charge of the positrons, it may be possible to mix the two species without adding energy to the antiprotons. Further theoretical and experimental studies are needed to evaluate the feasibility of utilizing the positron space charge for antiproton confinement with the goal of antihydrogen formation.

A limitation of the present work is that the time-dependent approach to an equilibrium isn't simulated. Only a final equilibrium is computed, and the theoretical treatment provides no assurance that there exists an experimental method for approaching the equilibrium. For approaching an equilibrium predicted here, it is envisioned that the system would first be prepared in the same way as is done for commencing a mixing experiment using the nested well approach to antihydrogen production. Next, the applied potentials would be changed to the same potential so as to remove the applied electric fields used to confine each species axially. The applied potentials would be switched on a timescale faster than other timescales, such that the positrons plasma is allowed to expand axially to the mirror coils. It is speculated that the positron plasma would begin to produce an axial potential energy well for antiprotons on a timescale that is sufficiently short for many antiprotons to remain trapped by the positron space charge. Throughout the procedure, two applied electric field regions could be maintained that would be located just beyond the magnetic mirror coils. The two applied electric field regions would serve for providing axial positron confinement after the positron plasma is allowed to expand axially.

After the positron plasma is allowed to expand axially, the positron plasma would be much longer than currently employed using the nested well approach to antihydrogen production. Confinement of such long positron plasmas may be an issue. Although it may be possible to construct an apparatus with a shorter distance between magnetic mirror coils, it may not be necessary. The positron plasma may only need to remain confined for a timescale somewhat longer than the three-body recombination timescale, once the positron plasma is allowed to expand axially. In fact, it may be desirable for the positron plasma to only remain confined for a timescale somewhat longer than the three-body recombination timescale, assuming that trappable antihydrogen would be produced primarily during a time period approximately equal to the three-body 
recombination timescale. Trapped antihydrogen that leaves the positron plasma could become ionized as a result of passing back through the positron plasma. Newly formed antiatoms that avoid being ionized by collisions with positrons as a result of traveling out of the positron plasma represent what may be observed experimentally as an enhancement of the three-body recombination rate, as compared to the rate predicted by a theoretical treatment that considers a plasma of infinite extent. Such a theoretical treatment leads to the $T^{9 / 2}$ temperature dependence of the timescale used in the present work, and much shorter timescales may occur experimentally due to the finite size of the positron plasma.

\section{Summary}

Electrostatic equilibria of a positron plasma in a model of the ALPHA trap were computed. The model consists of a uniform magnetic field with magnetic mirror fields superimposed and is radially bounded by grounded Malmberg-Penning trap electrodes. The transverse octupole field was assumed to be negligible compared to the axial field near the radial center of the trap where the plasma is located. The positron plasma was considered to extend to the mirror coils and follow the Boltzmann density distribution along magnetic field lines.

Computational techniques were developed to apply the Boltzmann density distribution in a non-uniform magnetic field. Equilibria were computed by solving Poisson's equation using a finite-difference method. The formation of an electrostatic potential well was found, and the possibility of using the potential well to confine an antiproton plasma was explored. The electrostatic potential well depths and plasma dimensions for a positron plasma and a two-species plasma were evaluated under various parameters. Timescales associated with three-body recombination, antiproton loss from the potential well due to antiproton-antiproton collisions, and temperature equilibration between the antiproton and positron plasmas were calculated. An equilibrium was found where the three-body recombination timescale is the smallest.

\section{Acknowledgments}

Computational resources were provided by UNT's High Performance Computing Services. This material is based upon work supported by the National Science Foundation under Grant No. PHY-1500427 and by the Department of Energy under Grant No. DE-FG02-06ER54883.

\section{Appendix}

The magnetic field everywhere in space of a circular loop of radius $R_{\mathrm{m}}$, carrying current $I_{\mathrm{m}}$ is (see, for example, [32])

$$
B_{\mathrm{m}, \mathrm{r}}(r, z)=\frac{\mu_{0} I_{\mathrm{m}}}{2 \pi R_{\mathrm{m}}} \alpha_{1}
$$




$$
\begin{aligned}
& \times\left(\beta_{1} \mathrm{E}(m)-\mathrm{K}(m)\right), \\
B_{\mathrm{m}, \mathrm{z}}(r, z) & =\frac{\mu_{0} I_{\mathrm{m}}}{2 \pi R_{\mathrm{m}}} \alpha_{2} \\
& \times\left(\beta_{2} \mathrm{E}(m)+\mathrm{K}(m)\right),
\end{aligned}
$$

where $\mu_{0}$ is the vacuum permeability, and $\mathrm{K}$ and $\mathrm{E}$ are the complete elliptic integrals of the first and second kind, respectively. The elliptic integrals and their argument are defined as

$$
\begin{aligned}
& \mathrm{K}(m)=\int_{0}^{\pi / 2} \frac{\mathrm{d} \theta}{\sqrt{1-m \sin ^{2} \theta}} \\
& \mathrm{E}(m)=\int_{0}^{\pi / 2} \mathrm{~d} \theta \sqrt{1-m \sin ^{2} \theta} \\
& m \quad \equiv \frac{4 R_{\mathrm{m}} r}{\left(r+R_{\mathrm{m}}\right)^{2}+z^{2}},
\end{aligned}
$$

and the dimensionless coefficients $\alpha_{1}, \beta_{1}, \alpha_{2}$, and $\beta_{2}$ are

$$
\begin{aligned}
\alpha_{1} & =\frac{R_{\mathrm{m}} z}{(r+\delta) \sqrt{\left(R_{\mathrm{m}}+r\right)^{2}+z^{2}}}, \\
\beta_{1} & =\frac{R_{\mathrm{m}}^{2}+r^{2}+z^{2}}{\left(R_{\mathrm{m}}-r\right)^{2}+z^{2}}, \\
\alpha_{2} & =\frac{R_{\mathrm{m}}}{\sqrt{\left(R_{\mathrm{m}}+r\right)^{2}+z^{2}}}, \\
\beta_{2} & =\frac{R_{\mathrm{m}}^{2}-r^{2}-z^{2}}{\left(r-R_{\mathrm{m}}\right)^{2}+z^{2}},
\end{aligned}
$$

where a constant with sufficiently small value to not disturb the calculations, $\delta=10^{-10}$, is added to remove the singularity at the origin.

\section{Works Cited}

[1] Andresen G B et al. 2010 Nature 468 673-6

[2] Andresen G B et al. 2008 J. Phys. B: At. Mol. Opt. Phys. 41011001

[3] Richerme P et al. 2013 Phys. Rev. A 87023422

[4] Gabrielse G et al. 2012 Phys. Rev. Lett. 108113002

[5] Widmann E et al. 2012 AIP Conf. Proc. 1441 546-8

[6] Lundmark R, Malbrunot C, Nagata Y, Radics B, Sauerzopf C and Widmann E 2015 J. Phys. B: At., Mol. Opt. Phys. 48184001

[7] Krasnicky D et al. 2013 AIP Conf. Proc. 1521 144-53

[8] Kellerbauer A et al. 2008 Nucl. Instrum. Methods B 266 351-6

[9] Perez P and Sacquin Y 2012 Class. Quantum Grav. 29184008

[10] Andresen G B et al. 2011 Nat. Phys. 7 558-64

[11] Amole C et al. 2012 Nature 483 439-43

[12] Zhmoginov A I, Charman A E, Shalloo R, Fajans J and Wurtele J S 2013 Class. Quantum Grav. 30205014

[13] Amole C et al. 2013 Nat. Comm. 41785

[14] Pritchard D E 1983 Phys. Rev. Lett. 51 1336-9

[15] Andresen G B et al. 2011 Phys. Lett. B 695 95-104 
[16] Andresen G B et al. 2011 Phys. Rev. Lett. 106025002

[17] Amole C et al. 2012 New J. Phys. 14015010

[18] Fajans J 2003 Phys. Plasmas 10 1209-14

[19] Gomberoff K, Fajans J, Wurtele J, Friedman A, Grote D P, Cohen R H and Vay J L 2007 Phys. Plasmas 14052107

[20] Spencer R L, Rasband S N and Vanfleet R R 1993 Phys. Fluids B 5 4267-72

[21] Ordonez C A 2003 Phys. Rev. E 67046401

[22] Ordonez C A and Weathers D L 2008 Phys. Plasmas 15083504

[23] Pacheco J L, Ordonez C A and Weathers D L 2012 Phys. Plasmas 19102510

[24] Berger M J and Oliger J 1984 J. Comp. Phys 53 484-512

[25] Amole C et al. 2014 Nucl. Instrum. Methods A 735 319-40

[26] Robicheaux F and Hanson J D 2004 Phys. Rev. A 69010701

[27] Chang Y, Correa J R and Ordonez C A 2004 Phys. Plasmas 11 3360-7

[28] Pastukhov V P 1974 Nuclear Fusion 14 3-6

[29] Ordonez C A and Molina M I 1994 Phys. Plasmas 1 2515-8

[30] Correa J R, Chang Y and Ordonez C A 2002 AIP Conf. Proc. 606 544-9

[31] Chang Y and Ordonez C A 2004 Phys. Rev. E 69037401

[32] Lane R A and Ordonez C A 2014 AIP Adv. 4077117 